\title{
Análise da resistência flexural de resinas acrílicas termopolimerizáveis utilizadas em placas oclusais
}

\author{
Analysis of flexural strength of acrylic resins with thermal polymerization \\ used in occlusal splint
}

Juliana Maria Porto Meireles de Andrade* Lúcia Helena Marques de Almeida Lima** Alcione Barbosa Lira de Farias ${ }^{* * *}$ Ana Isabella Arruda Meira Ribeiro**

Carmem Dolores de Sá Catão ${ }^{* * * *}$

George Carlos dos Santos Anselmo ${ }^{* * * *}$

\section{Resumo}

As resinas acrílicas são os materiais mais comumente empregados para a confecção de órteses oclusais, contudo, apresentam propriedades críticas no que tange à resistência e longevidade. Objetivo: o presente estudo buscou avaliar a resistência flexural de duas resinas acrílicas termopolimerizáveis empregadas em órteses oclusais: (Vipi Cril Plus ${ }^{\circledR}$ ) e (Vipi Wave ${ }^{\circledR}$ ). Materiais e método: 80 corpos-de-prova foram confeccionados e divididos de acordo com o método de polimerização: convencional e por micro-ondas. Os corpos-de-prova de cada grupo $(n=10)$ foram imersos em água destilada (grupo controle) e saliva artificial (grupo teste) e armazenados em estufa bacteriológica a $37^{\circ} \mathrm{C}$ durante os períodos de 7 e 15 dias. Posteriormente, as amostras foram submetidas ao ensaio mecânico de flexão em três pontos. Resultados: A análise estatística mostrou que houve diferença significativa $(p=0,02)$ entre os grupos, quando comparado o tipo de polimerização. Entretanto, não apresentou diferença estatística significativa entre as substâncias de armazenamento dos corpos-de-prova, bem como, também não revelou diferença quanto aos períodos de armazenagem. Conclusão: pode-se concluir que a polimerização por energia de micro-ondas apresentou maior resistência flexural do que a condição de polimerização convencional, não evidenciando influência da saliva artificial nem do tempo de imersão.

Palavras-chave: Resinas acrílicas. Placas oclusais. Polimerização.

\section{Introdução}

Disfunção temporomandibular (DTM) é um termo genérico que engloba várias condições de desordens da articulação temporomandibular desde alterações articulares, miofasciais até mesmo sensoriais $^{1,2}$. Essas disfunções são caracterizadas primeiramente por dor, ruídos articulares e funções irregulares ou limitadas da mandíbula ${ }^{3}$.

A DTM apresenta etiologia multifatorial, podendo estar associada a hábitos parafuncionais como o bruxismo do sono e/ou bruxismo em vigília 4 . Dentre as modalidades conservadoras e reversíveis de tratamento, tem-se o uso de placas oclusais ${ }^{5,6}$.

As placas oclusais, também chamadas de órteses oclusais, protetor de mordida, protetor noturno, placa interoclusal ou aparelho ortopédico, são dispositivos amplamente utilizados como tratamento coadjuvante dos sintomas da DTM, bem como, são recomendadas para pacientes que exibem alto índice de apertamento e bruxismo ${ }^{7}$.

As órteses oclusais rígidas podem ser confeccionadas em resina acrílica termopolimerizável ${ }^{8}$ (por polimerização convencional e por micro-ondas) ou autopolimerizável e com compósitos ${ }^{9}$.

Graduação em Odontologia - Universidade Estadual de Campina Grande (UEPB), PB, Brasil.

Professoras Doutoras do Departamento de Odontologia - Universidade Estadual de Campina Grande (UEPB), PB, Brasil.

Professora Mestre do Departamento de Odontologia - Universidade Estadual de Campina Grande (UEPB), PB, Brasil.

Professora Doutora do Departamento de Medicina e Enfermagem - Universidade Federal de Campina Grande (UFCG), PB, Brasil.

Doutorando em Ciências e Engenharia de Materiais pela Universidade Federal de Campina Grande (UFCG), PB, Brasil. 
Embora a resina acrílica seja o principal componente na confecção de placas oclusais, algumas apresentam fratura por queda do aparelho ou por fadiga do material diante do uso ${ }^{10}$. Um dos fatores relacionados à fratura é a resistência flexural que é a capacidade de um determinado material em resistir às forças que provocam a sua curvatura sem ser fraturado ou sofrer deformação excessiva ${ }^{11,12}$. Essa importante propriedade apresentada pelas resinas pode ser justificada pelo desenvolvimento inicial de microfraturas nas áreas onde se concentram as tensões até a ruptura completa do material ${ }^{13}$.

Dessa forma, considerando a relevância de estudos que abordem a longevidade das órteses oclusais empregadas no tratamento dos sintomas do bruxismo, o presente estudo se propôs a avaliar in vitro a resistência flexural de diferentes resinas acrílicas termicamente ativadas utilizadas na confecção desses dispositivos.

\section{Materiais e método}

Foram selecionados dois tipos de resinas acrílicas termicamente ativadas (RAAT), uma por polimerização através do método convencional (Vipi Cril Plus ${ }^{\circledR}$, Vipi Indústria, Comércio, Exportação e Importação de Produtos Odontológicos Ltda, Pirassununga, SP, Brasil) e outra por meio da energia de micro-ondas (Vipi Wave ${ }^{\circledR}$, Vipi Indústria, Comércio, Exportação e Importação de Produtos Odontológicos Ltda, Pirassununga, SP, Brasil).

A confecção e realização dos ensaios mecânicos dos espécimes foram realizadas seguindo os parâmetros determinados pela especificação $\mathrm{n}^{\mathrm{o}} 12$ da ADA (American Dental Association $)^{14}$ e pela norma para ensaios mecânicos ASTM D-790-10 (2010). Foram confeccionados 40 corpos de provas de cada resina acrílica, através de uma matriz em aço inoxidável no formato de barras retangulares, com arestas nítidas nas dimensões de $67 \mathrm{~mm} \times 12,5 \mathrm{~mm} \times 2,55$ $\mathrm{mm}$, resultando num total de 80 amostras.

As muflas metálicas para o ciclo de polimerização convencional e as de plástico reforçadas com fibras de vidro para o ciclo de polimerização por energia de micro-ondas foram previamente isoladas com vaselina sólida e preenchidas com gesso pedra tipo III (Herodent Soli-Rock ${ }^{\circledR}$, Vigodent, Rio de Janeiro, RJ, Brasil), sobre o qual foram posicionadas três matrizes metálicas com distanciamento de $1 \mathrm{~cm}$ entre cada uma delas.

Sobre as matrizes metálicas foi colocada silicona de condensação (Zetalabor ${ }^{\circledR}$ - Zhermack, Rovigo, Itália) para formar uma muralha e facilitar a confecção das amostras em resina acrílica.

Posteriormente, a contra mufla foi preenchida com o mesmo gesso pedra tipo III e submetida a uma carga de $1250 \mathrm{Kgf}$ na prensa hidráulica. Após a cristalização do gesso, a contra mufla foi removida ficando visível a cópia negativa das matrizes metálicas na silicona de condensação.

Feita a remoção destas barras metálicas, o gesso presente na mufla e contra mufla recebeu duas camadas de isolante para gesso (CEL LAC SS White Artigos Dentários Ltda, Rio de Janeiro, RJ, Brasil), em seguida, fez-se a manipulação da resina acrílica nas proporções especificadas pelo fabricante e os espaços obtidos sobre a silicona de condensação foram preenchidos com resina acrílica na fase plástica. Em seguida, a resina foi coberta por um filme plástico de polietileno de alta densidade e submetida a uma prensagem de forma lenta e gradual até se estabelecer uma pressão inicial de $750 \mathrm{Kgf}$ e final de 1000 Kgf. Após retirar o filme plástico e o excesso de material, a mufla foi fechada novamente e realizou-se a prensagem definitiva (1250 Kgf). Aguardou-se 20 minutos antes da polimerização.

Em seguida, a mufla foi levada à panela de alumínio e ao forno micro-ondas conforme o tipo de resina utilizada. Na polimerização convencional, a mufla foi acomodada, em uma panela com 3 litros de água fria, ligou-se o fogão com chama baixa por 30 minutos, então a chama foi aumentada e manteve-se a fervura por 90 minutos, em seguida, desligou-se a chama. Já para a polimerização por micro-ondas, a mufla foi acomodada em forno doméstico de micro-ondas com potência de $800 \mathrm{~W}$, por 20 minutos com 10/20\% de potência (baixa) e mais 5 minutos com $50 / 60 \%$ da potência (média). Aguardou-se o resfriamento completo das muflas para posterior abertura.

Após a demuflagem, os corpos-de-prova foram submetidos ao acabamento e polimento pelo método mecânico em lixadeira manual sob refrigeração constante, a uma sequência de três lixas d'água para polimento de diferentes granulações de carbeto de silício no ${ }^{\circ} 400,600$ e 1200 (Norton $^{\circledR}$, Saint-Gobain, França) em ordem crescente de granulações até atingirem as dimensões adequadas. Para o polimento final, foi usado um disco de feltro embebido em solução diamantada com granulometria de $1 \mu \mathrm{m}, 0,3 \mu \mathrm{m}$ e $0,05 \mu \mathrm{m}$ (Buehler), em uma polidora automática Politriz Universal APL-4 (Arotec ${ }^{\circledR}$, Cotia, SP, Brasil), durante um minuto, para cada solução. A aferição das dimensões foi realizada com paquímetro digital (Stainless Harddened Stainless Sales Corporation, Chicago, USA) até atingirem as dimensões de $65 \mathrm{~mm}$ de comprimento $10 \mathrm{~mm}$ de largura e 2,5 mm de espessura.

Posteriormente, os corpos de prova foram distribuídos equitativamente entre os grupos conforme a Tabela 1. 
Tabela 1 - Distribuição dos grupos de acordo com o tipo de resina, solução testada e tempo de armazenamento, onde (C) corresponde ao grupo controle e $(T)$ ao grupo teste

\begin{tabular}{|c|c|c|c|}
\hline Resina & Substância & Tempo (dias) & $(n=80)$ \\
\hline \multirow{4}{*}{ Vipi Cril Plus ${ }^{\circledR}$} & Água destilada (C) & 7 & 10 \\
\hline & Água destilada (C) & 15 & 10 \\
\hline & Saliva artificial* $(\mathrm{T})$ & 7 & 10 \\
\hline & Saliva artificial $(\mathrm{T})$ & 15 & 10 \\
\hline \multirow{4}{*}{ Vipi Wave $^{\circledR}$} & Água destilada (C) & 7 & 10 \\
\hline & Água destilada (C) & 15 & 10 \\
\hline & Saliva artificial $(\mathrm{T})$ & 7 & 10 \\
\hline & Saliva artificial (T) & 15 & 10 \\
\hline
\end{tabular}

${ }^{*}$ Composição da saliva artificial: Cloreto de potássio $(0,96 \mathrm{~g})$, Cloreto de sódio $(0,67 \mathrm{~g})$, Cloreto de Magnésio $(0,04 \mathrm{~g})$, Fosfato de Potássio (0,27 g), Cloreto de Cálcio $(0,12 \mathrm{~g})$, Nipagin $(0,01 \mathrm{~g})$, Nipasol $(0,1 \mathrm{~g})$, Carboxil Metil Celulose $(8 \mathrm{~g})$, Sorbitol $(24 \mathrm{~g})$, Água purificada q.s.p. (1000 mL).

Após o armazenamento nas respectivas soluções, as amostras foram colocadas em estufa bacteriológica a $37{ }^{\circ} \mathrm{C}$ durante o período de 7 e 15 dias, para posterior realização dos testes laboratoriais.

O ensaio mecânico para registro da resistência flexural e cálculo do módulo de elasticidade foi realizado através de um teste de carga em três pontos. Os testes foram executados através de uma máquina universal de ensaios Instron 5582 (Instron Ltda, Barueri, SP Brasil), obedecendo aos parâmetros estabelecidos pela norma ASTM D-790-10 (2010).

Ao final dos intervalos de tempo supracitados, os corpos-de-prova foram submetidos ao teste de ensaio de resistência flexural de três pontos continuamente até a fratura, por meio de dois apoios localizados a $50 \mathrm{~mm}$ um do outro e carga de flexão aplicada no centro do corpo-de-prova. O teste foi executado com o auxílio de uma célula de carga de $100 \mathrm{~N}$, partindo de 0 até $100 \mathrm{~N}$, com aumento gradativo de $5 \mathrm{~N}$, com uma pré-carga inicial de $0,5 \mathrm{~N}$. A velocidade do cabeçote foi constante em $5 \mathrm{~mm} / \mathrm{min}^{15}$.

Essa situação cria tensões de natureza complexa, com o início da fratura na zona de aplicação da carga e término na área de tração, com formação de microtrincas. Os corpos de prova foram submetidos à carga compressiva até a fratura. Aqueles que não sofreram a fratura, foram expostos à deformação até uma deflexão máxima registrada em milímetros pela máquina de teste, a partir da qual o ensaio era interrompido.

Os valores em MPa da resistência flexural $(\sigma)$ foram obtidos utilizando a seguinte fórmula:

$\sigma=3 F I / 2 b h^{2}$, onde $F$ é a carga máxima em Newton; $i$ a distância entre os suportes, isto é, $50 \mathrm{~mm} ; b$ a largura dos espécimes e $h$ a altura dos espécimes ${ }^{15}$.

Para se obter o valor em Newton suportado por cada corpo de prova, multiplicou-se o valor em Kgf registrado pela máquina Instron 5582 (Instron
Ltda. São Paulo, SP, Brasil) pelo valor aproximado da força de gravidade, ou seja, 9,807.

\section{Resultados}

Pôde-se perceber que a polimerização por micro-ondas apresentou maior média de resistência flexural no $7^{\circ}$ dia tanto para a condição de saliva artificial, quanto para a condição de água destilada (Tab. 2). Esse padrão não foi observado na polimerização convencional, pois, se na condição de saliva artificial o $7^{\circ}$ dia apresentou maior média de resistência, para a condição de água destilada, a maior média de resistência foi para o $15^{\circ}$ dia.

Foi encontrado, após a realização da Análise de Variância Fatorial (ANOVA), um efeito estatisticamente significativo quanto ao tipo de polimerização na resistência flexural das resinas $(p<0,02)$ (Tab. 3). Assim como, foi verificada uma diferença estatisticamente significante da resistência entre os métodos de polimerização (Tab. 4), mostrando que a polimerização por energia de microondas apresentou maior média que a condição de polimerização convencional (Fig. 1).

Tabela 2 - Média da resistência flexural dos corpos de prova polimerizados por energia de micro-ondas e pelo método convencional, submetidos ao armazenamento nos intervalos de 7 e 15 dias em saliva artificial e água destilada

\begin{tabular}{l|c|c|c|c}
\hline \multirow{2}{*}{ Acrilização } & Substância & Tempo & Média & $\begin{array}{c}\text { Desvio } \\
\text { padrão }\end{array}$ \\
\hline \multirow{3}{*}{ Micro-ondas } & Saliva Artificial & 7 dias & 86,06 & 14,74 \\
& & 15 dias & 80,79 & 6,49 \\
& \multirow{2}{*}{ Água destilada } & 7 dias & 84,08 & 9,25 \\
& \multirow{2}{*}{ Convencional dias } & 78,15 & 9,62 \\
\cline { 3 - 5 } & \multirow{2}{*}{ Saliva artificial } & 7 dias & 77,56 & 14,27 \\
& & 15 dias & 73,79 & 16,07 \\
& Água destilada & 7 dias & 73,63 & 12,26 \\
& & 77,32 & 17,08 \\
\hline
\end{tabular}


Tabela 3 - Análise de Variância Fatorial quanto à resistência flexural para estudo da correlação entre as variáveis

\begin{tabular}{l|c|c|c}
\hline \multicolumn{1}{c|}{ Fonte } & $F$ & Sig. & Eta parcial quadrado \\
\hline Acrilização & 5,10 & 0,02 &, 068 \\
Saliva & 0,18 & 0,67 &, 003 \\
Tempo & 0,91 & 0,34 &, 013 \\
Acrilização * Saliva & 0,12 & 0,72 &, 002 \\
Acrilização * Tempo & 0,88 & 0,35 &, 012 \\
Saliva * Tempo & 0,33 & 0,56 &, 005 \\
Acrilização * Saliva * Tempo & 0,47 & 0,49 &, 007 \\
\hline
\end{tabular}

Tabela 4 - Teste t-Student para verificar a existência de diferença estatística quanto à resistência flexural entre os métodos de polimerização estudados

\begin{tabular}{c|c|c|c|c|c}
\hline $\mathrm{t}$ & Sig. & Diferença & Erro padrão & \multicolumn{2}{|c}{$95 \%$ Intervalo de confiança } \\
\cline { 5 - 6 } & média & de diferença & Inferior & Superior \\
\hline 2,28 & 0,02 & 6,59 & 2,89 & 0,83 & 12,35 \\
\hline
\end{tabular}

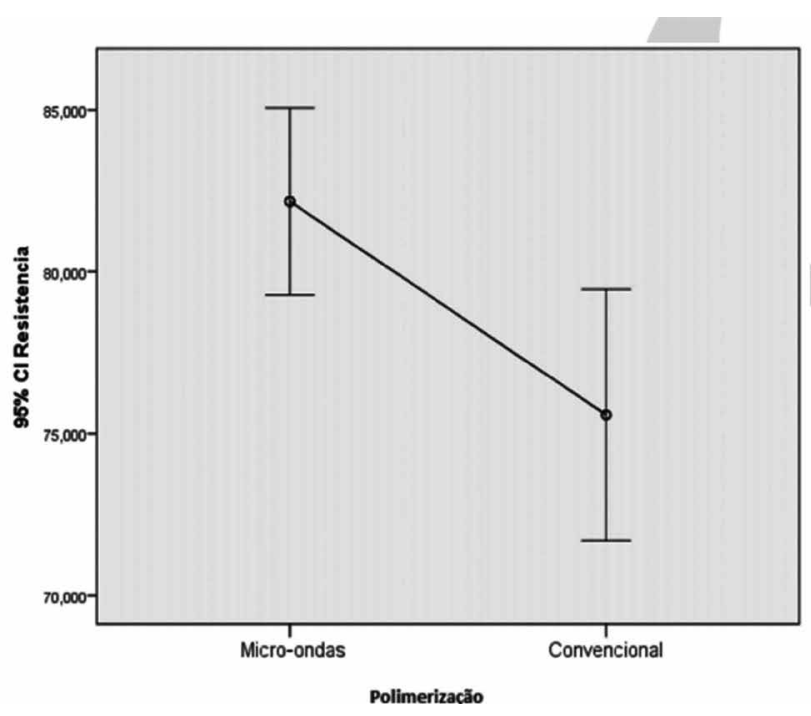

Figura 1 - Intervalos de confiança entre as condições de polimerização

\section{Discussão}

As resinas acrílicas são compostas pelo pó de polimetilmetacrilato (PMMA) e monômero de metilmetacrilato (MMA) ${ }^{16}$. Desde o seu surgimento, há mais de 60 anos, continua sendo o material mais utilizado para a confecção de bases protéticas e órteses oclusais por apresentar qualidades estéticas, facilidade de manipulação ${ }^{17}$, bom ajuste e polimento ${ }^{18}$.

O conhecimento das propriedades físicas e químicas das resinas acrílicas empregadas na odontologia foi fundamental para o estabelecimento da Especificação n ${ }^{\circ} 12$ para polímeros acrílicos da American Dental Association ${ }^{14}$ utilizada neste trabalho.

Posteriormente, a possibilidade da utilização do calor seco, proporcionado pela energia gerada pelas micro-ondas para a polimerização das resinas acrílicas, levou ao desenvolvimento de um tipo específico de resina. O tempo de processamento laboratorial reduzido, quando empregado este método, assume um caráter diferencial importante na escolha desta técnica, uma vez que não ocorre perda das propriedades físicas, químicas e mecânicas consideradas necessárias a uma prótese adequada ${ }^{19}$.

Dessa forma, a polimerização por microondas está sendo indicada como uma solução rápida e adequada em comparação à polimerização tradicional em água ${ }^{20}$. Com o intuito de analisar a propriedade mecânica da RAAT por polimerização de micro-ondas, empregou-se a resina Vipi Wave ${ }^{\circledR}$ neste estudo.

A variação do tempo de polimerização e da potência das micro-ondas aplicadas às resinas acrílicas foi estudada por Ilbay et al. ${ }^{21}$ (1994) e Botega et al. ${ }^{22}$ (2004) que buscaram encontrar padrões adequados de polimerização. A correta utilização desta energia depende do tempo e da potência do apare$l^{23}{ }^{23}$, a maioria dos autores emprega 3 minutos à potência de $500 \mathrm{~W}^{24,25}$. Blagojevic e Murphy ${ }^{26}$ (1999) polimerizaram as resinas pelo mesmo tempo, porém à potência de $600 \mathrm{~W}$.

A polimerização das RAAT por microondas, empregadas nesse estudo, obedeceu ao ciclo sugerido pelo fabricante da resina Vipi Wave ${ }^{\circledR}$. Esse fato pode ter influenciado na maior conversão ou polimerização dessa resina.

A resistência flexural corresponde a uma propriedade mecânica dos materiais, normalmente avaliada por meio de um teste de carga de três pontos, que dá valores às cargas suportadas por esses materiais submetidos à flexão até a sua fratura ou deformação permanente ${ }^{27}$. Esse teste mensura simultaneamente a tensão, isto é, força sobre unidade de área, de tração, de compressão e de cisalhamento de um corpo de prova em forma de barra quando submetido a uma carga. A modificação resultante na barra é representada por uma deformação compressiva na região superior e uma tração (alongamento) na região inferior ${ }^{28}$. Em corpos finos o suficiente, como os que foram utilizados neste trabalho, há um predomínio de tensões de tração ao longo da superfície inferior.

A resistência flexural e o módulo de elasticidade da resina são influenciados pelo grau de conversão alcançado com a sua técnica de polimerização ${ }^{29}$. Quanto maior o grau de conversão dos monômeros em polímeros, maior a resistência da resina. Nesta pesquisa, o resultado apontou uma diferença significativa $(p=0,02)$ de média de 6,59 entre os métodos empregados, evidenciando que a técnica por energia de micro-ondas apresentou maior resistência que a convencional.

Ilbay et al. ${ }^{21}$ constataram que a resina polimerizada por energia de micro-ondas foi mais resistente a falhas mecânicas do que a polimerizada pelo mé- 
todo convencional, corroborando os resultados deste estudo. Entretanto, discordaram dos achados de Levin et al. ${ }^{29}$ (1989) que não encontraram diferença significativa em relação a porosidade, dureza e resistência à flexão de resinas acrílicas polimerizadas convencionalmente e por micro-ondas.

Braun et al. ${ }^{30}(2000)$, avaliando a alteração dimensional em resinas polimerizadas pelo método convencional e por energia de micro-ondas, observaram alterações semelhantes nas duas técnicas, enquanto Goiato et al. ${ }^{31}$ (2009) não relataram nenhuma alteração dimensional em seus estudos. Embora não tenham sido avaliadas as alterações dimensionais das resinas, neste trabalho, parece que o comportamento dimensional pode estar relacionado a um maior número de poros, o que pode favorecer a introdução de água dentro das cadeias poliméricas.

Os resultados desta pesquisa contrapõem os achados de Rached e Del Bel Cury ${ }^{32}$ (2001) e Silva et al. ${ }^{33}$ (2002), nos quais observou-se ausência de influência desses fatores na resistência à flexão apresentada pelas resinas acrílicas convencionais e para micro-ondas, assim como aos achados de Koumjian e $\mathrm{Nimmo}^{34}$, que não encontraram diferenças na resistência flexural de amostras após o armazenamento em água a $37^{\circ} \mathrm{C}$ durante 7 dias, quando comparado com o armazenamento em ambiente seco a $23{ }^{\circ} \mathrm{C}$ no mesmo tempo.

Em relação à resistência flexural da RAAT convencional, percebeu-se um decréscimo na condição de armazenamento em saliva artificial entre os intervalos de 7 e 15 dias e um discreto aumento quando armazenada em água destilada. Esse aumento encontrado concordou com os achados de Maekawa et al. ${ }^{35}$ em que observou-seque as médias de resistência aumentaram com o decorrer do tempo de imersão em água destilada e saliva artificial a uma temperatura de $37^{\circ} \mathrm{C}$.

A resina polimerizada, quando imersa, absorve água e essa quantidade de embebição, embora não muito grande, resulta em um intumescimento que ocorre na sua maior parte, após duas semanas de imersão em água destilada à temperatura de $37{ }^{\circ} \mathrm{C}^{36}$. O intumescimento é causado pela introdução de água na massa polimerizada que pode interferir nas ligações cruzadas da cadeia polimérica e, portanto, alterar as características do polímero resultante ${ }^{27}$. A ocorrência de tal efeito é caracterizada por uma maior mobilidade entre as cadeias do polímero, fazendo com que a água absorvida ocupe espaços entre essas cadeias ${ }^{37}$.

O resultado médio de resistência flexural da RAAT por micro-ondas encontrado foi semelhante aos demonstrados por alguns autores como Kounjian e $\mathrm{Ninmo}^{34}(1990), 76,81 \mathrm{MPa}$, Ogawa et al. ${ }^{38}$ (2000), 73,00 MPa, Haselton et al. ${ }^{39}$ (2002), 97,90 MPa.
Algumas diferenças encontradas entre este estudo e os registrados na literatura podem ser justificadas por diversos fatores, pois há algumas diferenças na composição entre os sistemas de resina PMMA disponíveis na Odontologia, tanto no mercado nacional quanto em outros países, além de várias técnicas de processamento, desde a proporção pó:líquido até a polimerização e armazenamento.

A importância da proporção pó:líquido está no efeito direto sobre a quantidade de monômero residual formado pela massa polimérica final ${ }^{27}$, uma maior concentração deste monômero pode interferir negativamente na resistência flexural pela formação de porosidades internas que concentram tensões quando essa resina é submetida a uma carga qualquer ${ }^{27,28}$.

Os resultados deste estudo corroboraram os achados de Smith et al. ${ }^{40}$ (1992) nos quais observou-seque as RAAT por energia de micro-ondas apresentaram maior dureza e menor flexibilidade que as convencionais. É preciso considerar a realização de futuros estudos que abordem a análise microestrutural das resinas acrílicas tanto após a polimerização, quanto em diferentes períodos e substâncias de armazenamento, além de verificar a densidade dos corpos de prova e classificar as superfícies de fratura.

\section{Conclusão}

Os resultados deste estudo mostraram que a polimerização das RAAT por energia de micro-ondas apresentou maior resistência flexural do que a técnica convencional. Entretanto, não foram evidenciadas influências da imersão em saliva artificial nem do tempo de estocagem nesta propriedade.

\section{Abstract}

Acrylic resins are the most commonly used materials in the fabrication of occlusal splints, however they present critical properties in terms of strength and longevity. Objective: this study sought to assess the flexural strength of two acrylic resins with thermal polymerization used in occlusal orthotics: (Vipi Cril Plus ${ }^{T M}$ ) and (Vipi Wave ${ }^{T M}$ ). Materials and method: 80 test samples were prepared and divided according to the polymerization method: conventional and microwave. The specimens of each group $(n=10)$ were immersed in distilled water (control group) and artificial saliva (test group), and stored in a bacteriological oven at $37{ }^{\circ} \mathrm{C}$ for perio$d s$ of 7 and 15 days. Subsequently, the samples were subjected to mechanical bending tests on three points. Results: Statistical analysis showed a significant difference $(p=0.02)$ between the groups, when compared to the type of polymerization. However, there was no significant statistical difference between the substances of specimens' storage, and there was also no difference regarding storage periods. Conclusion: It was possible to conclude that polymerization by microwave energy 
showed higher flexural strength than the conventional polymerization condition, showing no influence of artificial saliva or immersion time.

Keywords: Acrylic resins. Occlusal splints. Polymerization.

\section{Referências}

1. La Touche R, Fernández-De-Las-Peñas C, Fernández-Carnero J, Escalante k, Angulo-Díaz-Parreño S, Paris-Alemany A, et al. The effects of manual therapy and exercises directed at the cervical spine on pain sensitivity in patients with myofascial temporomandibular disorders. J Oral Rehabil 2009; (36):644-52.

2. Nixdorf DR, John MT, Schierz O, Bereiter DA, Hellenkant G. Self-repoted severity of taste disturbances correlates with dysfunctional grade of TMD pain. J Oral Rehabil 2009; (36):792-800.

3. Oliveira VW. Disfunções temporomandibulares. v. 6. São Paulo: Artes Médicas; 2002.

4. Okeson, JP. Tratamento das Desordens Temporomandibulares e Oclusão. 7. ed. São Paulo: Elsevier; 2013.

5. Alencar Júnior FGP, Aizawa AS, Camparis CM. Placas oclusais e suas indicações no tratamento de pacientes com disfunção craniomandibular (DCM). JBC J Bras Odontol Clín 1998; 2(11):56-62.

6. Issa JPM, Silva MAMR, Silva AMBR. Disfunções temporomandibulares e suas implicações clínicas. Rev Dor 2005; $6(2): 573-8$

7. Portero PP, Kern R, Kusma SZ, Grau-Grullón P. Placas oclusais no tratamento da disfunção temporomandibular (DTM). Rev Gestão \& Saúde 2009; 1(1):36-40.

8. Soboleva U, Jokstad A, Eckersberg T, Dahl Bl. Chewing movements in TMD patients and a control group before and after use of a stabilization splint. Int J Prosthodont 1998; $11(2): 158-64$

9. Leib AM. Patient preference for light-cured composite bite splint compared to heat-cured acrylic bite splint. J Periodontol 2001; 72(8):1108-12.

10. Yamamoto ETC, Uemura ES, Maekawa MY, Bagni BA, Rosa RGS, Destro ASS. Avaliação da resistência flexural de resinas acrílicas polimerizadas por dois métodos. RSBO 2009; 6(2):148-54.

11. Silva LH, Tango RN, Kimpara ET, Saavedra GSFA, Paes-Junior TJA. Resistência à flexão e microdureza da resina acrílica quimicamente ativada submetida a tratamento por energia de microondas. Rev Gaúcha Odontol 2011; 59(2):237-42.

12. Xible AA, Tavarez RRJ, Araújo CPR, Bonachela WC. Effect of silica coating and silanization on flexural and compositeresin bond strengths of zircônia posts: an in vitro study. J Prosthet Dent 2006; 95(3):224-9.

13. Soares RG, Botelho AL, Cecchin D, Cruvinel DR, Catuse ABCEB, Pagnano VO. Resistência flexural e rugosidade superficial de resinas acrílicas utilizadas para confecção de placas oclusais. Rev Fac Odontol Univ Passo Fundo 2008; 13(3):60-5.

14. American Dental Association. Specifications $\mathrm{n}^{\circ} 12$ for denture base polymers. Councils on dental materials and devices, reports of councils and bureaus. J Am Dent Assoc 1975; (90):39-49.
15. Bastos LGC. Avaliação da resistência flexional, do módulo de elasticidade e do tipo de fratura de uma resina acrílica para restaurações provisórias-efeito de diversos tipos de reforços. [Dissertação de Mestrado]. Bauru: Faculdade de Odontologia de Bauru da Universidade de São Paulo; 2003.

16. Rantala LI, Lastumaki TM, Pelotomaki T, Vallitu PK. Fatigue resistance of removable orthodontic appliance reinforced with glass fiber weave. J Oral Rehabil 2003; (30):501-6.

17. Straioto FG, Ricomini Filho AP, Fernandes Neto AJ, Del Bel Cury AA. Polytetrafluorethylene added to acrylic resins: Mechanical properties. Braz Dent J 2010; 21(1):55-9.

18. Koroglu A, Ozdemir T, Usanmaz A. Comparative study of the mechanical properties of fiber reinforced denture base resin. J Appl Polym Sci 2009; 113:716-20.

19. Phoenix RD. Resinas para base de prótese total. In: Anusavice KJ. Phillips Materiais Dentários. 11.ed. Rio de Janeiro: Elsevier Editora Ltda; 2005. p. 679-713.

20. Duarte ARC, Ayb EA, Bonachela WC, Salvador MCG, Viera LF. Avaliação de rugosidade superficial e resistência flexional de quatro resinas acrílicas utilizadas para próteses sobre implante, em função imediata. UFES Rev Odontol 2006; $8(1): 37-45$

21. Ilbay SG, Gunever S, Alkumuru HN. Procesing dentures using microwave techinique. J Oral Rehabil 1994; 21(1):103-9.

22. Botega DM, de Souza Machado T, de Melo JÁ, Garcia RC, Del Bel Cury AA. Polymerization time for a microwave-cured resin with multiple flasks. Braz Oral Res 2004; 18(1):23-8.

23. Santos PH, Gomes EA, Pavan S,Vergari CE. Energia por microondas: efeito na estabilidade dimensional de resinas acrílicas. Rev Odont Univ C 2007; 19(1):84-9.

24. Salim S, Sadamori S, Hamada T. The dimensional accuracy of retangular acrylic resin specimens cured by three denture bases processing methods. J Prosthet Dent St. Louis 1992; 67(6):879-81

25. Turck MD, Richards MW. Microwave processing for denture relines, repairs, and rebases. J Prosthet Dent St. Louis 1993; 69(3):340-3.

26. Blagojevic V, Murphy VM. Microwave polymerization of denture base materials. A comparative study. J Oral Rehabil Oxford 1999; 26(10):804-8.

27. Anusavice KJ. Materiais dentários. 10. ed. Rio de Janeiro: Guanabara Koogan; 2000.

28. Donovan TE, Turst RG, Campagni WV. Phisical properties of acrylic polymerized by different techniques. J Prosthet Dent $1985 ; 54(4): 522-4$.

29. Levin B, Sanders JL, Reitz PV. The use of microwave Energy for processing acrylic resins. J Prosthet 1989; 61(3):381-3.

30. Braun KO, Rodrigue Garcia RCM, Rizzatti-Barbosa CM, Del Cury AA. Alteração dimensional linear de resinas para bases de próteses polimerizadas com microondas. Pesqui Odontol $2000 ; 14(3): 278-82$.

31. Goiato MC, Santos DM, Gennari Filho H, Zavanelli AC, Dekon SFC, Mancuso DN. Influence of investment, disinfection and storage on the microhardness of ocular resins. J Prosthodontics 2009; 18(1):32-5.

32. Rached RN, Del Bel Cury AA. Heat-cured resin repaired with microwave-cured on: bond strength and surface texture. J Oral Rehabil 2001; 28(4):370-5. 
33. Silva FA, Silva TB, Rached RN, Del Bel Cury. Effect of intrinsic pigmentation on the fleural strengtn of mizowave-cured acrylic resin. Braz Dent J 2002; 13(3):205-7.

34. Koumjian JH, Nimmo A. Evaluation of fracture resistance of resins used for provisional restorations. J Prosthet Dent $1990 ; 64: 654-7$

35. Maekawa MY, Uemura ES, Borges ALS, Otari ER, Maekawa LE, Marcacci S. Estudo do efeito da saliva artificial na resistência flexural de resinas acrílicas para prótese. Rev Odont Univ SP 2006; 18(2):2161-6.

36. Braden M. The absorption of water by acrylic resins and other materials. J Prosthet Dent 1964; 14(12):307-16.

37. Pero AC, Barbosa DB, Marra J, Ruvolo-Filho AC, Compagnoli MA. Influence of microwave polymerization method and thickness on porosity of acrylic resin. J Prosthodont 2008; $17: 125-9$.

38. Ogawa T, Tanaka M, Koyano K. Effect of water temperature during polymerization on strength of auto polymerizing resin. J Prosthet Dent 2000; 84(2):222-4.

39. Haselton DR, Diaz-Arnold AM, Vargas MA. Flexural strength of provisional crown and fixed partial denture resins. Prosthet Dent 2002; 87(2):225-8.

40. Smith LT, Powers JM, Ladd D. Mechanical properties of new denture resins polymerized by visible light, heat, and microwave energy. Int J Prosthodont 1992; 5(4):315-20.

\section{Endereço para correspondência:}

Ana Isabella Arruda Meira Ribeiro

BR 104, KM 119, S/N, Condomínio Nações

Residence Privê

Quadra F, Lote 11, CEP 58117-000 Lagoa Seca - PB

Fone: (83) 87900118 ou (83) 33373649

E-mail: isaro_jesus@hotmail.com

Recebido: 09/11/2013. Aceito: 08/05/2014. 